\title{
Função pulmonar em portadores de anemia falciforme
}

\author{
Lung function in patients with sickle cell anemia
}

\begin{abstract}
Cássia Suzane V. Fonseca1, Carlos André Araújo-Melo², Ricardo Matias de Carvalho², José Barreto-Neto², Jerônimo Gonçalves Araújo ${ }^{4}$, Rosana Cipolotti ${ }^{5}$
\end{abstract}

\section{RESUMO}

Objetivo: Avaliar a função ventilatória por meio de espirometria, em escolares e adolescentes com anemia falciforme $(\mathrm{AF})$, relacionando os achados a parâmetros clínicos e hematológicos.

Métodos: Foram avaliados portadores de AF de ambos os gêneros, a partir dos dez anos, clinicamente estáveis, sem intercorrências agudas, que foram submetidos à espirometria e avaliados quanto à saturação transcutânea de oxigênio, níveis de hemoglobina e contagem de leucócitos. Verificou-se a associação de alterações à espirometria com as características demográficas, clínicas e laboratoriais dos pacientes analisados. Para a análise estatística, aplicou-se o teste do qui-quadrado e o teste $t$ para amostras não pareadas, sendo significante $p<0,05$.

Resultados: Foram estudados 51 pacientes e, em 40 (78\%), identificou-se comprometimento do perfil espirométrico, do quais $20(50 \%)$ apresentaram distúrbio ventilatório misto ou combinado, 13 (33\%) mostraram perfil restritivo clássico e sete (18\%), distúrbio ventilatório obstrutivo. Dos sete, em cinco $(71 \%)$ observou-se resposta broncodilatadora positiva. A contagem total de leucócitos associou-se à função pulmonar alterada. O volume expiratório forçado no primeiro segundo sem broncodilatador, a capacidade vital forçada antes e após broncodilatador e o fluxo expiratório forçado entre 25 e $75 \%$ da capacidade vital forçada após broncodilatador foram significativamente menores nos pacientes com relato de internação hospitalar prévia por doença pulmonar aguda.
Conclusões: A maioria dos pacientes apresentou alteração da função pulmonar, predominando o padrão misto ou combinado, seguido pelo restritivo clássico. Presença de leucocitose, na ausência de intercorrências agudas, associouse a comprometimento de função pulmonar.

Palavras-chave: anemia falciforme; pulmão; espirometria; Pediatria.

\section{ABSTRACT}

Objective: To evaluate the pulmonary function in children and adolescents with sickle cell disease (SCD) and to associate the findings with clinical and hematologic characteristics of the studied population.

Methods: Male and female SCD patients with ten or more years old, clinically stable and without acute clinical problems were tested by spirometry. At that time, total pulse oximetry values, hemoglobin and total white blood cell count were verified. Association between altered pulmonary function tests and demographic, clinical and laboratorial characteristics of the patients were assessed. Statistical analysis included chi-square and t-test, being significant $p<0.05$.

Results: Among the 51 studied patients, $40(78 \%)$ had abnormal spirometric results: 20 (50\%) had mixed or combined ventilatory disorders, $13(33 \%)$ had classical restrictive profile and seven (18\%), obstructive ventilatory disorder. Out of these seven patients, five $(71 \%)$ had a positive response to bronchodilators. Increased total white
Instituição: Universidade Federal de Sergipe (UFS), São Cristóvão, SE, Brasil ${ }^{1}$ Médica Radiologista do Hospital Universitário da UFS, São Cristóvão, SE, Brasil

${ }^{2}$ Médico do Hospital Universitário da UFS, São Cristóvão, SE, Brasil

${ }^{3}$ Doutor em Pneumologia pela Universidade Federal de São Paulo; Professor colaborador do Departamento de Medicina da UFS, São Cristóvão, SE, Brasil

${ }^{4}$ Mestre em Ciências da Saúde pela UFS; Professor Assistente do Departamento de Medicina da UFS, São Cristóvão, SE, Brasil

${ }^{5}$ Doutora em Pediatria/Hematologia pela Faculdade de Medicina de Ribeirão Preto da Universidade de São Paulo; Professora Adjunta do Departamento de Medicina da UFS, São Cristóvão, SE, Brasil
Endereço para correspondência:

Rosana Cipolotti

Avenida Beira Mar, 2.016, apto. 402

CEP 49025-040 - Aracaju/SE

E-mail: rosanaci@yahoo.com

Fonte financiadora: Programa Institucional de Bolsas de Iniciação Científica do Conselho Nacional de Desenvolvimento Científico e Tecnológico.

Conflito de interesse: nada a declarar

Recebido em: 23/11/2009

Aprovado em: 25/6/2010 
blood cells count was associated with abnormal pulmonary function. Patients with previous acute pulmonary hospital admissions had lower forced expiratory volume in one second without bronchodilators, lower forced vital capacity before and after bronchodilators, and lower forced expiratory flow between 25 and $75 \%$ of the forced vital capacity after bronchodilators.

Conclusions: Most SCD patients presented abnormal pulmonary function, being predominant the mixed or combined type, followed by the classical restrictive pattern. Increased white blood cell count in the blood, without acute clinical events, was associated to abnormal pulmonary function.

Key-words: anemia, sickle cell; lung; spirometry; Pediatrics.

\section{Introdução}

A anemia falciforme (AF) é uma hemoglobinopatia de caráter genético, descrita pela primeira vez em $1910^{(1)}$. No Brasil, os dados sobre a prevalência da doença apontam uma frequência expressiva, ainda que baseada em estudos regionais ${ }^{(2)}$. Caracteriza-se clinicamente por comprometimento multissistêmico, resultando em morbidade e mortalidade ${ }^{(3)}$. A ocorrência de fenômenos vasoclusivos representa o evento fisiopatológico determinante, causando isquemia, disfunção endotelial, proliferação vascular, inflamação e estresse oxidativo. Esses mecanismos resultam em vasculopatia proliferativa, que pode afetar as circulações cerebral, renal e, inclusive, pulmonar ${ }^{(4)}$.

Estudos anteriores ${ }^{(5)}$ identificaram alterações crônicas na função pulmonar dos portadores de AF, independentemente do antecedente de síndrome torácica aguda (STA), porém não contemplaram a faixa etária dos adolescentes. Os adultos com AF têm função pulmonar comprometida, mais frequentemente assumindo as características de distúrbio ventilatório restritivo (DVR) $)^{(6-8)}$, resultante de repetidos episódios de vaso-oclusão, infecção e embolia gordurosa, os quais têm início ainda na infância ${ }^{(6,9,10)}$. Complicações pulmonares são a principal causa de morbimortalidade entre portadores de AF; manifestam-se, em geral, na $2^{\text {a }}$ década de vida e culminam com óbito na $4^{a}$ década. Lesões pulmonares provocadas por obstrução de via aérea superior, infecções pulmonares repetidas e estado pró-inflamatório crônico levam a distúrbio ventilatório obstrutivo ou restritivo, resultando em hipertensão pulmonar e morte. Um estudo multicêntrico cita mais de $20 \%$ de complicações pulmonares fatais em adultos ${ }^{(11)}$. Anormalidades na função pulmonar também foram descritas em crianças pequenas, nas quais o padrão restritivo torna-se mais proeminente com o avanço da idade, fator possivelmente associado à hipertensão pulmonar e à hipoxemia crônica do adulto. $\mathrm{O}$ desenvolvimento da hipertensão pulmonar é outro fator que aumenta a mortalidade ${ }^{(9,10)}$.

As provas de função pulmonar parecem ser um dos primeiros testes a se alterarem no curso da doença pulmonar e, assim, devem ser realizadas em todos os portadores de AF. A espirometria é um exame de execução fácil, de baixo custo e razoavelmente sensível para detectar e confirmar a doença pulmonar, principalmente em indivíduos sob risco. Este estudo objetivou avaliar a função ventilatória em escolares e adolescentes com AF, utilizando-se a espirometria como ferramenta diagnóstica, e verificar a associação de resultados anormais com alguns parâmetros clínicos e de exames complementares, empregados na avaliação de rotina desses pacientes.

\section{Método}

Foi realizado um estudo entre julho e setembro de 2009 com pacientes de ambos os gêneros, idade a partir dos dez anos e diagnóstico de AF comprovado por eletroforese de hemoglobina, acompanhados no Serviço de Hematologia Pediátrica do Hospital Universitário da Universidade Federal de Sergipe há, pelo menos, 12 meses. Os critérios de exclusão foram presença de cardiopatia congênita ou adquirida não relacionada à $\mathrm{AF}$ e de asma em uso de corticosteroide ou broncodilatador.

Os responsáveis pelos pacientes elegíveis foram convidados a participar após apreciação e assinatura de termo de consentimento livre e esclarecido. As informações referentes a sintomas respiratórios (tosse, "falta de ar", "dor no peito") durante o mês que antecedeu a consulta e diagnóstico anterior de asma ou alergia respiratória em qualquer época da vida foram obtidas em entrevista com os responsáveis pelos pacientes. Dados referentes à internação anterior por síndrome torácica aguda foram obtidos dos prontuários.

Foram avaliadas a frequência cardíaca e a saturação transcutânea de oxigênio por meio de oxímetro portátil (modelo 9500 Nonin Onyx, Nonin Medical Inc., Plymouth, MN, USA), com medidas em intervalo de um ponto percentual. De cada paciente, foram tomadas três medidas em cada consulta (no início do atendimento, antes 
do exame físico e após entrega da prescrição) em três consultas eletivas consecutivas, na ausência de intercorrências ou queixas agudas. O sensor foi posicionado no segundo quirodáctilo esquerdo e foi calculada a média aritmética dos valores obtidos. Foram obtidos também os valores médios da hemoglobina e leucócitos totais nas mesmas três consultas consecutivas.

As espirometrias foram sempre realizadas no mesmo período do dia e pela mesma técnica. Utilizou-se espirômetro acoplado a um computador modelo Spiro USB (Micro Medical Ltd., United Kingdom) para determinar as curvas volume-tempo e fluxo-volume. Os pacientes foram orientados a permanecer sentados durante a realização dos testes e a utilizar o clipe nasal. Foram realizados testes de broncodilatação ( $400 \mathrm{mcg}$ de sulfato de salbutamol em spray). Três curvas foram realizadas, obedecendo aos critérios de aceitabilidade e reprodutibilidade da American Thoracic Society ${ }^{(12)}$, sendo escolhida a melhor delas. A partir dessa curva, foram computados os valores da capacidade vital forçada (CVF), volume expirado forçado no primeiro segundo (VEF1) e fluxo expiratório forçado entre 25 e $75 \%$ da CVF (FEF25-75\%), todos corrigidos para as condições BTPS (Body Temperature and Pressure Satured). Os resultados das provas de função pulmonar foram analisados por um mesmo pneumologista com treinamento e experiência na realização de espirometria em escolares, adolescentes e adultos, sendo comparados a padrões de referência estabelecidos ${ }^{(13,14)}$

Os resultados obtidos foram analisados por meio do software Statistical Package for the Social Sciences (SPSS) 13.0 para Windows. As variáveis foram expressas em proporções, com intervalo de confiança de $95 \%$. As proporções entre grupos foram comparadas por meio do teste do qui-quadrado e as médias, pelo teste $t$ de Student para amostras não pareadas. O nível de significância adotado foi de $5 \%(p<0,05)$.

O projeto da pesquisa foi aprovado pelo Comitê de Ética em Pesquisa em Seres Humanos da Universidade Federal de Sergipe como parte do projeto temático "Estudos Clínicos em Doença Falciforme”.

\section{Resultados}

Foram estudados 51 pacientes, com idade média de $15 \pm 4$ anos (variação: 10-26), dos quais $57 \%$ eram do gênero masculino. $\mathrm{O}$ sintoma atual mais comum foi tosse e a duração dos sintomas foi de 18 dias em média. Em 57\% dos pacientes estudados houve referência a dois ou mais episódios de doença pulmonar aguda prévia, com necessidade de internação hospitalar, dos quais $12 \%$ tinham registro em prontuário de internação por síndrome torácica aguda no ano anterior. Somente dois pacientes tinham diagnóstico prévio de asma, mas nenhum usava terapia de manutenção (Tabela 1).

A saturação média de oxigênio por oximetria transcutânea foi de $93,7 \pm 4,5 \%$ (variação: $84-100$ ) e a frequência cardíaca média foi de $93 \pm 14$ batimentos por minuto (variação: 56-120). A contagem leucocitária total média foi de 10.979 \$3.959 leucócitos $/ \mathrm{mm}^{3}$ (variação: 6.250-17.000) e o valor médio de hemoglobina foi de $8,6 \pm 1,6 \mathrm{~g} / \mathrm{dL}$ de sangue (variação: 6,2-12,2).

Os testes de função pulmonar aplicados nos 51 pacientes estudados resultaram nos seguintes valores, descritos em média e desvio padrão: volume expiratório forçado no primeiro segundo (VEF1) sem broncodilatador: 1,85 $\pm 0,65 \mathrm{~L}$; VEF1 após broncodilatador 1,86 $\pm 0,64 \mathrm{~L}$; capacidade vital forçada (CVF) sem broncodilatador: 1,99 $\pm 0,75$ L; CVF após broncodilatador 2,03 $\pm 0,73 \mathrm{~L}$; fluxo expiratório forçado entre 25 e $75 \%$ da capacidade vital forçada (FEF 25-75\%) sem broncodilatador: 2,39 $\pm 0,90 \mathrm{~L} / \mathrm{s}$; FEF25-75\% após broncodilatador: 2,61 $\pm 0,92 \mathrm{~L} / \mathrm{s}$. Ou seja, dentre os 51 pacientes, $40(78,4 \%)$ apresentaram prova de função respiratória alterada e, destes, 50\% (20 pacientes) apresentaram distúrbio ventilatório misto (DVM). Em 32,5\% ( $\mathrm{n}=13)$, foi observado distúrbio ventilatório restritivo (DVR) clássico e, em 17,5\% $(\mathrm{n}=7)$, distúrbio ventilatório obstrutivo (DVO). Desses sete pacientes, cinco $(71,4 \%)$ tiveram resposta broncodilatadora positiva. Os valores do VEF1 pré-broncodilatador (BD), CVF pré e pós-BD e FEF25-75\% pós-BD foram significativamente menores naqueles com relato de doença pulmonar aguda prévia (Tabela 2).

Não houve diferença estatisticamente significante nos parâmetros espirométricos quando os pacientes foram alocados segundo o nível de hemoglobina. Quando as crianças e os adolescentes foram divididos de acordo com o nível de leucócitos, com o ponto de corte de $15 \mathrm{mil} / \mathrm{mm}^{3}$, observou-se que o valor do FEF25-75\% pós-BD foi significantemente menor no grupo de pacientes com valor igual ou superior a $15 \mathrm{mil} / \mathrm{mm}^{3}$. Os valores de VEF1 pré e pós-BD, CVF pré e pós-BD e FEF25-75\% pré-BD mostram tendência a associarem-se à contagem de leucócitos, mas sem significância estatística (Tabela 3). Nenhuma diferença relevante foi notada quando os pacientes foram estratificados de acordo com a saturação transcutânea de oxigênio, e também não houve diferença entre os parâmetros analisados, segundo o padrão ventilatório. 
Tabela 1 - Características dos pacientes portadores de anemia falciforme conforme o perfil à espirometria

\begin{tabular}{|c|c|c|c|c|}
\hline & Total $(n=51)$ & $\begin{array}{l}\text { Espirometria } \\
\text { normal }(n=11)\end{array}$ & $\begin{array}{l}\text { Espirometria } \\
\text { alterada }(n=40)\end{array}$ & Valor de $p$ \\
\hline Idade (anos)* & $14,8(10-26)$ & 14,0 & 14,9 & 0,48 \\
\hline Sexo masculino & $29(57 \%)$ & $6(55 \%)$ & $23(58 \%)$ & 0,99 \\
\hline Não branco & $44(86 \%)$ & $11(100 \%)$ & $33(83 \%)$ & 0,32 \\
\hline DPAP com IH & & & & 0,41 \\
\hline - Nenhum episódio & $15(29 \%)$ & $5(45 \%)$ & $10(25 \%)$ & \\
\hline - 1 episódio & $7(14 \%)$ & $1(10 \%)$ & $6(15 \%)$ & \\
\hline - $\geq 2$ episódios & $29(57 \%)$ & $5(50 \%)$ & $24(59 \%)$ & \\
\hline Tosse & $32(63 \%)$ & $6(55 \%)$ & $26(65 \%)$ & 0,73 \\
\hline Dor no peito & $7(14 \%)$ & $3(27 \%)$ & $4(10 \%)$ & 0,16 \\
\hline Falta de ar & $5(10 \%)$ & $1(9 \%)$ & $4(10 \%)$ & 0,99 \\
\hline Duração sintomas (dias) $)^{\star *}$ & 17,8 & $14,7 \pm 20,3$ & $18,6 \pm 13,3$ & 0,54 \\
\hline Alergia respiratória & $12(24 \%)$ & $4(36 \%)$ & $8(20 \%)$ & 0,26 \\
\hline Asma & $2(4 \%)$ & $1(9 \%)$ & $1(3 \%)$ & 0,39 \\
\hline STA no ano anterior & $6(12 \%)$ & $2(18 \%)$ & $4(10 \%)$ & 0,58 \\
\hline $\mathrm{SpO}_{2}$ ambulatorial $^{* *}$ & $93,7 \pm 4,5$ & $93,1 \pm 3,6$ & $93,9 \pm 4,7$ & 0,65 \\
\hline FC ambulatorial** & $93,0 \pm 14,2$ & $93,2 \pm 14,3$ & $92,9 \pm 14,3$ & 0,95 \\
\hline $\mathrm{Hb}$ ambulatorial** & $8,6 \pm 1,6$ & $8,0 \pm 0,8$ & $8,8 \pm 1,8$ & 0,14 \\
\hline GB ambulatorial** & $10.979 \pm 3.950$ & $10.206 \pm 3.270$ & $13.791 \pm 5.023$ & 0,006 \\
\hline
\end{tabular}

*Mediana (variação); **média \pm desvio padrão; DPAP com IH: doença pulmonar aguda prévia com internação hospitalar; STA: síndrome torácica aguda; $\mathrm{SpO}_{2}$ saturação transcutânea de oxigênio (\%); FC: frequência cardíaca (bpm); Hb: hemoglobina (g/dL); GB: contagem total de glóbulos brancos (/dL).

Tabela 2 - Valores dos parâmetros ventilatórios dos portadores de anemia falciforme em média \pm desvio padrão, conforme antecedente de doença pulmonar aguda

\begin{tabular}{lccc}
\hline & \multicolumn{2}{c}{ Doença pulmonar aguda anterior } & \multirow{2}{*}{ Valor de $\boldsymbol{p}$} \\
\cline { 2 - 3 } & Não $(\mathbf{n}=\mathbf{1 5})$ & $\operatorname{Sim}(\mathbf{n}=\mathbf{3 6})$ & 0,03 \\
\hline VEF1 pré-BD & $2,04 \pm 0,79$ & $1,65 \pm 0,47$ & 0,06 \\
VEF1 pós-BD & $2,04 \pm 0,78$ & $1,71 \pm 0,47$ & 0,02 \\
CVF pré-BD & $2,25 \pm 0,91$ & $1,78 \pm 0,54$ & 0,03 \\
CVF pós-BD & $2,28 \pm 0,88$ & $1,84 \pm 0,55$ & 0,06 \\
FEF 25-75\% pré-BD & $2,65 \pm 1,09$ & $2,18 \pm 0,67$ & 0,02 \\
FEF 25-75\% pós-BD & $2,94 \pm 1,13$ & $2,36 \pm 0,64$ & \\
\hline
\end{tabular}

BD: broncodilatador; VEF1: volume expiratório forçado no primeiro segundo; CVF: capacidade vital forçada; FEF 25-75\%: fluxo expiratório forçado entre 25 e $75 \%$ da capacidade vital forçada.

\section{Discussão}

Entende-se desde 1886, a partir do trabalho de John Hutchinson ${ }^{(15)}$, que medidas espirométricas devem ser analisadas em comparação com valores obtidos para uma população padrão e que fatores como idade, altura, gênero e etnia podem ter influência nos resultados ${ }^{(16)}$. Entretanto, os resultados são muito semelhantes entre 15 e 25 anos, exceto para grandes variações de estatura. O presente estudo incluiu pacientes a partir dos dez anos, porque já foi demonstrado que, em indivíduos com idade superior a nove anos, os dados espirométricos podem ser interpretados com os mesmos critérios 
Tabela 3 - Valores dos parâmetros ventilatórios dos portadores de anemia falciforme, conforme a contagem total de glóbulos brancos

\begin{tabular}{|c|c|c|c|}
\hline & \multicolumn{2}{|c|}{ Doença pulmonar aguda anterior } & \multirow{2}{*}{ Valor de $p$} \\
\hline & $<15.000 / \mathrm{mm}^{3}(\mathrm{n}=45)$ & $\geq 15.000 / \mathrm{mm}^{3}(\mathrm{n}=6)$ & \\
\hline VEF1 pré-BD & $1,88 \pm 0,67$ & $1,38 \pm 0,26$ & 0,08 \\
\hline CVF pré-BD & $2,05 \pm 0,77$ & $1,48 \pm 0,29$ & 0,08 \\
\hline CVF pós-BD & $2,10 \pm 0,77$ & $1,49 \pm 0,25$ & 0,05 \\
\hline FEF $25-75 \%$ pós-BD & $2,71 \pm 0,93$ & $1,89 \pm 0,17$ & 0,04 \\
\hline
\end{tabular}

BD: broncodilatador; VEF1: volume expiratório forçado no primeiro segundo; CVF: capacidade vital forçada; FEF 25-75\%: fluxo expiratório forçado entre 25 e $75 \%$ da capacidade vital forçada.

utilizados em adultos ${ }^{(16)}$. Em estudo com delineamento semelhante ${ }^{(5)}$, no qual foram avaliados 53 pacientes com AF, aqueles com idade entre 15 e 24 anos apresentaram maior porcentagem de resultados normais $(62,5 \%)$ e os $37,5 \%$ restantes exibiram padrão restritivo; já metade dos pacientes com idade superior a 24 anos mostrou padrão restritivo. No presente estudo, o distúrbio ventilatório misto foi o padrão de alteração da função pulmonar mais observado, sendo os pacientes mais jovens do que os estudados por Enright et al ${ }^{(16)}$.

O predomínio do distúrbio ventilatório misto neste estudo pode ser justificado pelo espectro clínico que caracteriza a AF, envolvendo anemia crônica, infartos pulmonares e presença de substâncias mediadoras da resposta inflamatória. Os transtornos ventilatórios restritivos identificados na população avaliada provavelmente se devem à ocorrência de infartos pulmonares causados por vaso-oclusão e pela embolia pulmonar gordurosa, que pode se seguir a episódios de isquemia óssea, com consequente substituição do parênquima pulmonar por tecido fibrótico. Esses eventos afetam a elasticidade pulmonar e a expansão da parede torácica, ocasionando as limitações observadas $^{(5,9)}$, situação que piora com a idade ${ }^{(9)}$. Note-se que entre os 11 pacientes com laudos espirométricos normais, apenas quatro possuíam idade superior a 15 anos. Deve-se lembrar que o padrão espirométrico de restrição pulmonar também pode ser resultado de inspiração ineficaz causada por dor e pelo comprometimento estrutural da caixa torácica em consequência de infartos das costelas durante o crescimento, de osteoporose ou osteomalácia vertebral ${ }^{(17)}$.

Entretanto, o padrão restritivo não é o único reflexo do comprometimento de função pulmonar que se verifica em longo prazo nos portadores de AF. A hipertensão pulmonar, cuja etiologia parece ser multifatorial, vem sendo reconhecida com frequência crescente em portadores dessa anemia hemolítica ${ }^{(4)}$. Estudos que observaram frequência elevada de padrão obstrutivo sugeriram que a recorrência da STA pode contribuir para distúrbios obstrutivos ${ }^{(18)}$ e que a hiper-reatividade brônquica é a principal causa da alteração observada ${ }^{(6)}$. Outro estudo cita o estado pró-inflamatório, que leva à leucocitose, ao aumento de moléculas de adesão e à elevação das citocinas, como provável agente causal da obstrução reversível das vias aéreas inferiores ${ }^{(4)}$. No entanto, a patogenia da hiper-reatividade brônquica não é completamente compreendida ${ }^{(19)}$.

Um estudo realizado na Jamaica ${ }^{(20)}$ sugere que a administração de broncodilatadores é útil no tratamento da STA em pessoas com AF, pois considera que a hiper-responsividade brônquica pode ser um componente da síndrome, mas tal orientação desperta controvérsias ${ }^{(19)}$. Ainda que, no presente estudo, as espirometrias tenham sido realizadas ambulatorialmente, em crianças e adolescentes sem evidências de STA, cinco dos sete pacientes que apresentaram DVO tiveram resposta positiva ao broncodilatador.

Estudos anteriores identificaram associação entre diagnóstico prévio de asma e padrão ventilatório obstrutivo com STA e crises dolorosas ${ }^{(6,8,10)}$. Pesquisas futuras poderão esclarecer a relação entre os achados de padrão ventilatório restritivo ou misto na infância e adolescência e a gravidade do comprometimento da função pulmonar no adulto portador de AF.

Alguns autores consideram que a oximetria de pulso é pouco específica para determinar a real saturação de oxigênio; entretanto, recomendam o uso dessa ferramenta para triagem de casos sob risco de alteração crônica da função pulmonar $^{(7)}$. O presente estudo não encontrou associação entre as alterações da espirometria e os valores de saturação percutânea de oxigênio.

Os achados aqui apresentados referem-se à avaliação transversal da função pulmonar de uma coorte de adolescentes e adultos jovens portadores de AF em acompanhamento 
prospectivo, com a finalidade de melhor caracterizar a gravidade das alterações e as repercussões em médio e longo prazo, bem como analisar o papel desempenhado por algumas medidas terapêuticas, destacando-se o uso de broncodilatadores, corticosteroides inalatórios, hemotransfusão de substituição e hidroxiureia. Conclui-se, portanto, que o distúrbio ventilatório misto foi o padrão espirométrico mais frequentemente observado nos pacientes avaliados. Para definir a relação entre o padrão espirométrico disfuncional, variáveis constitucionais e ambientais específicas de portadores de AF e o tipo e o grau de comprometimento da função pulmonar em longo prazo, é necessário o acompanhamento longitudinal dessa coorte.

\section{Referências bibliográficas}

1. Gómez-Chiari M, Puigbert JT, Aramburu JO. Drepanocitosis: experiência de um centro. An Pediatr 2003;58:95-9.

2. Silla LM. Doença falciforme: um grave e desconhecido problema de saúde pública no Brasil. J Pediatr (Rio J) 1999;75:145-6.

3. Organización Mundial de La Salud. $5^{\text {a }}$ Asamblea Mundial de La Salud. Punto 11.4 del orden del día provisional. Anemia Falciforme. Informe de la Secretaria. 24 de abril de 2006.

4. Machado RFP. Hipertensão arterial pulmonar associada à anemia falciforme. J Bras Pneumol 2007;33:583-91.

5. Mesa Cuervo JR, Chagéz Leyva O, Hechavarria Miyares J, Placencia Ternblóm A, Losada Buchillón R, Rodríguez LR et al. Modificaciones funcionales ventilatorias en pacientes con anemia drepanocítica y antecedentes de síndrome torácico agudo. Rev Cuba Hematol Inmunol Hemoter 2002;18.

6. Koumbourlis AC, Hurlet-Jensen A, Bye MR. Lung function in infants with sickle cell disease. Pediatr Pulmonol 1997;24:277-81.

7. Souza LC, Viegas CA. Quality of sleep and pulmonary function in clinically stable adolescents with sickle cell anemia. J Bras Pneumol 2007;33:275-81.

8. Powars D, Weidman JA, Odom-Maryon T, Niland JC, Johnson C. Sickle cell chronic lung disease: prior morbidity and the risk of pulmonary failure. Medicine (Baltimore) 1988;67:66-76.

9. Sylvester KP, Patey RA, Milligan P, Dick M, Rafferty GF, Rees D et al. Pulmonary function abnormalities in children with sickle cell disease. Thorax 2004;59:67-70.

10. Greenough A. Sickle cell disease - pulmonary complications and a proinflammatory state? Am J Respir Crit Care Med 2004;169:663-5.
11. Platt OS, Brambilla DJ, Rosse WF, Milner PF, Castro O, Steinberg MH et al. Mortality in sickle cell disease. Life expectancy and risk factors for early death. N Engl J Med 1994;330:1639-44.

12. Autoria não referida. Lung function testing: selection of reference values and interpretative strategies. American Thoracic Society. Am Rev Respir Dis 1991;144:1202-18.

13. Pereira CA. Valores de referência para a espirometria em uma amostra da população brasileira adulta [tese de doutorado]. São Paulo (SP): Unifesp; 1992.

14. Knudson RJ, Lebowitz MD, Holberg CJ, Burrows B. Changes in the normal maximal expiratory flow-volume curve with growth and aging. Am Rev Respir Dis 1983;127:725-34.

15. Hutchinson J. On the capacity of the lungs, and on the respiratory functions, with a view of establishing a precise and easy method of detecting disease by the spirometer. Med Chir Trans 1846;29:137-252.

16. Enright PL, Linn WS, Avol EL, Margolis HG, Gong H Jr, Peters JM. Quality of spirometry test performance in children and adolescents: experience in a large field study. Chest 2000;118:665-71.

17. Moreira GA. Repercussões respiratórias da anemia falciforme. J Bras Pneumol 2007;33:18-20.

18. Santoli F, Zerah F, Vasile N, Bachir D, Galacteros F, Atlan G. Pulmonary function in sickle cell disease with or without acute chest syndrome. Eur Respir J 1998;12:1124-9.

19. Heredia Rubio CD. Complicaciones pulmonares de la drepanocitosis. An Pediatr 2005;62:12-7.

20. Knight-Madden JM, Forrester TS, Lewis NA, Greenough A. Asthma in children with sickle cell disease and its association with acute chest syndrome. Thorax 2005;60:206-10. 